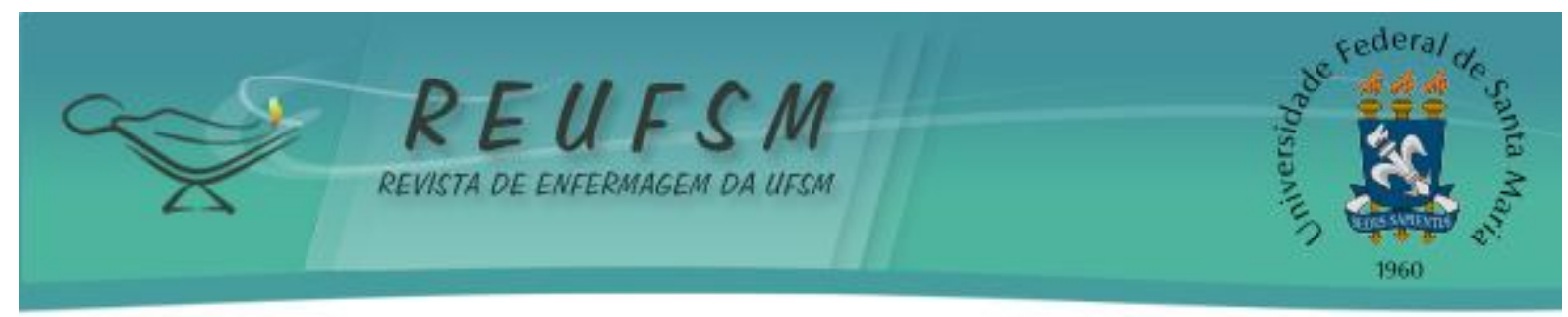

ARTIGO ORIGINAL

\title{
ASSISTÊNCIA DE ENFERMAGEM A PORTADORES DE FERIDAS: TECNOLOGIAS DE CUIDADO DESENVOLVIDAS NA ATENÇÃO PRIMÁRIA
}

\section{NURSING ASSISTANCE TO WOUNDS BEARERS: TECHNOLOGIES OF CARE DEVELOPED IN THE PRIMARY ATTENTION}

\section{ASISTENCIA DE ENFERMERÍA A LOS PORTADORES DE HERIDAS: TECNOLOGÍAS DE CUIDADO DESARROLLADAS EN LA ATENCIÓN PRIMARIA}

\author{
Josefine Busanello ${ }^{1}$ \\ Fernanda Machado da Silva ${ }^{2}$ \\ Graciela Dutra Sehnem ${ }^{3}$ \\ Marcia Adriana Poll ${ }^{4}$ \\ Luise Monteiro Lobão de Deus ${ }^{5}$ \\ Thyelle da Silva Bohlke
}

RESUMO: Objetivo: conhecer as tecnologias de cuidado implementadas no cuidado a indivíduos portadores de feridas em unidades de atenção primária de um município do interior do Rio Grande do Sul. Método: os sujeitos do estudo foram oito enfermeiros atuantes nessas unidades de saúde. A coleta de dados foi realizada no mês de julho de 2012, utilizando a técnica de grupo focal. Resultados: a partir da análise temática foram estruturadas três categorias: comunicação e vínculo na mediação do cuidado aos indivíduos portadores de feridas; sistematização da assistência de enfermagem aos indivíduos portadores de feridas; e terapias tópicas no cuidado aos indivíduos portadores de feridas. Considerações finais: no âmbito dos cuidados de enfermagem aos indivíduos portadores de feridas é imprescindível a implementação das tecnologias leves, leve-duras e duras. Todavia, as tecnologias leves precisam predominar, considerando a perspectiva representada pela atenção primária.

Descritores: Desenvolvimento tecnológico; Cuidados de enfermagem; Atenção primária à saúde; Cicatrização.

ABSTRACT: Aim: to know the care technologies implemented in the care of individuals with wounds in primary care units of a city in the interior of Rio Grande do Sul. Method: the study subjects were eight nurses working in these health units. Data collection was conducted in July 2012, using the focus group technique. Results: from the analysis were structured three thematic categories: Communication and relationship mediation care to individuals with wounds; systematization of nursing care to individuals with wounds, and topical therapies in the care of individuals with wounds. Final considerations: the scope of nursing care to individuals with

\footnotetext{
${ }^{1}$ Enfermeira. Doutora em Enfermagem. Professora Adjunta do Curso de Enfermagem da Universidade Federal do Pampa. Líder do Grupo de Estudos e Pesquisa em Enfermagem da Fronteira Oeste do Rio Grande do Sul (GEPEnf-FORS). E-mail: josefinebusanello@unipampa.edu.br

${ }^{2}$ Enfermeira. Mestre em Enfermagem. Professora Assistente do Curso de Enfermagem da Universidade Federal do Pampa. Pesquisadora do GEPEnf-FORS. E-mail: fernandadasi@yahoo.com.br ${ }^{3}$ Enfermeira. Mestre em Enfermagem. Professora Assistente do Curso de Enfermagem da Universidade Federal do Pampa. Pesquisadora do GEPEnf-FORS. E-mail: graci_dutra@yahoo.com.br ${ }^{4}$ Enfermeira. Mestre em Enfermagem. Professora Assistente do Curso de Enfermagem da Universidade Federal do Pampa. Pesquisadora do GEPEnf-FORS. E-mail: adripoll@hotmail.com

${ }^{5}$ Acadêmica do $8^{\circ}$ semestre do Curso de Enfermagem da Universidade Federal do Pampa. Integrante do GEPEnf-FORS. E-mail: luise1933@hotmail.com

${ }^{6}$ Acadêmica do $6^{\circ}$ semestre do Curso de Enfermagem da Universidade Federal do Pampa. Integrante do GEPEnf-FORS. E-mail: thyelle_idf@hotmail.com
} 


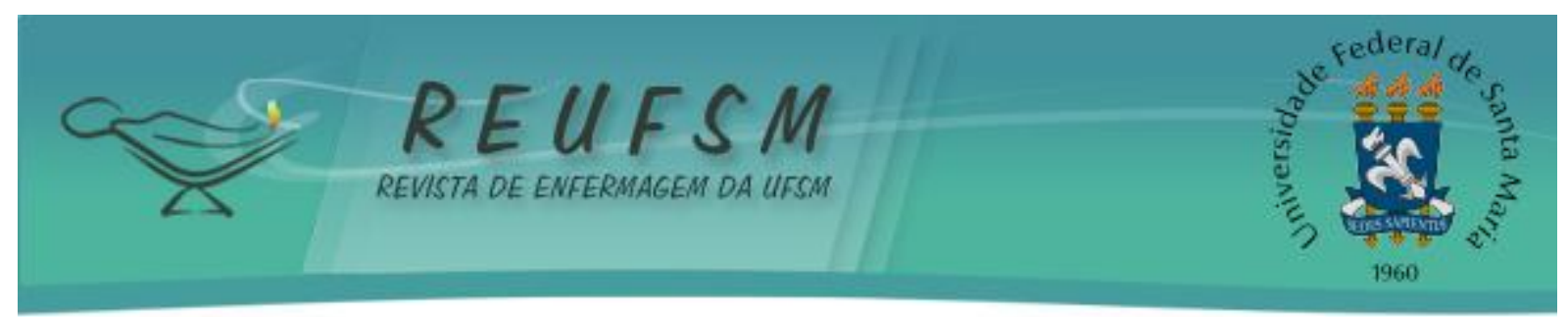

wounds is essential to the implementation of soft technologies, soft-hard and hard. However, the need to dominate light technologies, considering the perspective represented by primary care.

Descriptors: Technological development; Nursing care; Primary health care; Wound healing.

RESUMEN: Objetivo: conocer las tecnologías de atención implementadas en la atención de personas con lesiones en las unidades de atención primaria de una ciudad del interior de Rio Grande do Sul. Método: los sujetos de estudio fueron ocho enfermeros que trabajan en estas unidades de salud. La recolección de datos llevó a cabo en julio de 2012, mediante la técnica del grupo focal. Resultados: los resultados del análisis temático se estructuraron en tres categorías: comunicación y vinculo en la mediación de la atención a personas con heridas; sistematización de los cuidados de enfermería a personas con heridas, y terapias tópicas en la atención de personas con heridas. Consideraciones finales: en el ámbito de los cuidados de enfermería a personas con heridas es esencial para la implementación de tecnologías leves, leve-dura y dura. Sin embargo, la necesidad de dominar las tecnologías leves, teniendo en cuenta la perspectiva representada por la atención primaria.

Descriptores: Desarrollo tecnológico; Atención de enfermería; Atención primaria de salud; Cicatrización de heridas.

\section{INTRODUÇÃO}

O cuidado de enfermagem a portadores de feridas precisa contemplar ações voltadas para as dimensões biológicas, sociais e psicológicas desses indivíduos, não se restringindo somente a lesão cutânea. É preciso considerar o paciente em toda sua integralidade, da área lesionada até os fatores sistêmicos e psicossociais que podem alterar o processo de cicatrização. Assim, para atender todas as necessidades de saúde e de doença do indivíduo portador de feridas, torna-se imprescindível a utilização de tecnologias que contemplem a integralidade do cuidado de enfermagem.

As tecnologias de cuidado são definidas como um conjunto de conhecimentos científicos, empíricos e sistematizados, que estão em constante processo de inovação nos processos de trabalho em saúde. ${ }^{1}$ Essa perspectiva remete a ideia de que a instituição das tecnologias de cuidado tem como finalidade provocar intervenções sobre uma determinada situação de saúde ou de doença, a partir do desenvolvimento de um conjunto de conhecimentos para a construção de produtos materiais ou inter-relacionais. ${ }^{1}$

Historicamente, a discussão acerca desse conceito, iniciou no século XIX, com a primeira revolução técnico-científica, cujas transformações repercutiram intensamente em todos os mecanismos de produção. ${ }^{2}$ A partir desse movimento social e político, as tecnologias passaram a permear todos os âmbitos do desenvolvimento humano. No contexto da atenção em saúde, o termo tecnologia foi resgatado para fundamentar a mediação entre a racionalidade e a subjetividade, fazendo da razão e da sensibilidade instrumentos para fortalecer e qualificar as ações de cuidado.

Nesse sentido, as tecnologias se tornaram instrumentos que passaram a embasar ações abstratas ou concretas, com a finalidade de produzir o cuidado no processo de trabalho em saúde. ${ }^{3} \mathrm{O}$ cuidado de enfermagem, na perspectiva da tecnologia, contempla a capacidade do ser humano em buscar inovações, visando a qualidade e a resolutividade da atenção em saúde, mediante a inter-relação pessoal, teorias, métodos e processos científicos. ${ }^{4}$

As tecnologias de cuidados envolvidas no trabalho da enfermagem podem ser classificadas em leves, leve-duras e duras. As tecnologias leves permitem a produção de relações envolvidas no encontro entre o trabalhador da saúde e o usuário, mediante a escuta, o interesse e a construção de vínculos. É nesse território que a ética do exercício profissional e os saberes sobre a relação interpessoal adquirem importância. As tecnologias 


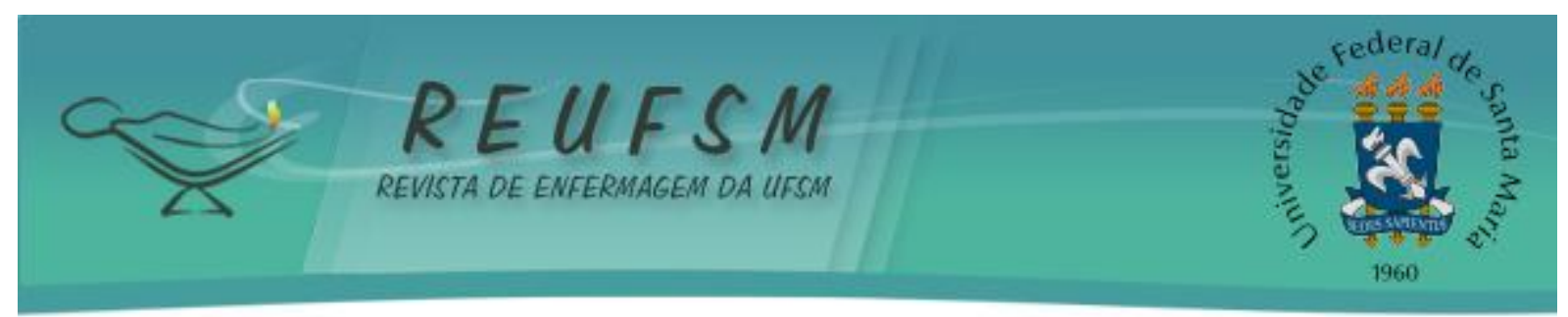

leve-duras permitem processar o olhar do profissional da saúde sobre o usuário, como um objeto de sua intervenção, a partir do raciocínio clínico e da epidemiologia. ${ }^{5}$

As tecnologias duras de cuidado envolvem equipamentos e estruturas organizacionais. Os equipamentos se constituem como dispositivos que possibilitam acessar dados físicos, exames laboratoriais e imagens, e medicamentos utilizados nas intervenções terapêuticas, sendo estes necessários para alimentar o raciocínio clínico e qualificar o cuidado em saúde. As estruturas organizacionais dos serviços de saúde são garantidas a partir do estabelecimento de normas e metodologias assistenciais, que direcionam a sistematização do cuidado. ${ }^{5}$

$\mathrm{Na}$ prática assistencial, a qualidade do cuidado de enfermagem pode ser alcançada a partir da combinação dessas tecnologias, o que determina os diferentes modelos de atenção à saúde. ${ }^{5}$ Considerando o processo de trabalho da enfermagem, evidencia-se que diferentes tecnologias são empregadas, buscando responder às exigências sociais e de saúde de determinada época e de certo espaço social. ${ }^{3}$

A atenção primária é um cenário propício para a implementação das tecnologias de cuidado de enfermagem ao indivíduo portador de ferida, pois é possível apreender a realidade de vida da população, o que favorece o processo de identificação dos problemas de saúde e de doença, e das alternativas para solucioná-los. Essa configuração viabiliza um conjunto de ações de saúde voltadas para os indivíduos e sua coletividade, tendo como finalidade a promoção e a manutenção da saúde, a prevenção de agravos, o diagnóstico, o tratamento e a reabilitação. ${ }^{6}$ Assim, questiona-se: Quais as tecnologias de cuidado implementadas pelos enfermeiros na atenção primária em saúde aos indivíduos portadores de feridas?

0 interesse em realizar esta pesquisa embasa-se na importância de abordar as tecnologias do cuidado dos indivíduos portadores de feridas, e na escassez de produções cientificas que contemplem essa temática. Ademais, cabe destacar que a presente pesquisa faz parte das ações de ensino, pesquisa e extensão desenvolvidas pelos docentes e acadêmicos do Curso de Enfermagem da Universidade Federal do Pampa, com o intuito de abordar os cuidados de enfermagem ao portador de feridas e o trabalho dos Enfermeiros e demais trabalhadores de Enfermagem que atuam no cuidado a esses indivíduos, nos diferentes serviços de saúde.

Diante do exposto, o presente estudo teve como objetivo conhecer as tecnologias de cuidado implementadas no cuidado a indivíduos portadores de feridas em unidades de atenção primária de um município do interior do Rio Grande do Sul.

\section{MÉTODO}

Esse estudo faz parte da macropesquisa intitulada Percepção de enfermeiros acerca dos cuidados de enfermagem a indivíduos portadores de feridas: perspectiva da Atenção Primária do interior do Rio Grande do Sul. Teve uma abordagem qualitativa, de caráter exploratório. ${ }^{7}$ As Unidades Básicas de Saúde (UBS) de um município do interior do Rio Grande do Sul foram os cenário investigativos da pesquisa.

Os sujeitos foram oito enfermeiros atuantes nessas unidades de saúde. 0 critério de seleção dos participantes foi: estar participando do curso de aperfeiçoamento sobre cuidados de enfermagem a indivíduos portadores de feridas, desenvolvido, paralelamente, ao projeto da macropesquisa.

A coleta de dados foi realizada no mês de julho de 2012, a partir da técnica de grupo focal. 0 grupo focal é um tipo de entrevista em grupo que valoriza a comunicação entre os participantes da pesquisa, com o objetivo de gerar dados e valorizar a interação grupal para prover tipos distintos de dados. ${ }^{8}$ Os encontros focais, com duração de aproximadamente duas horas, foram conduzidos por um moderador e um observador não participante, em ambiente adequado. 


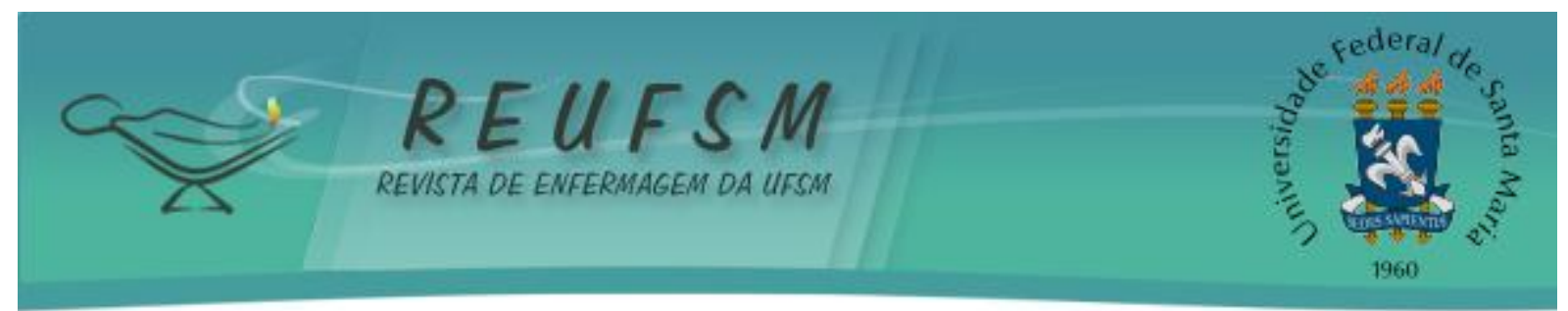

Especificamente, para o presente estudo, foram considerados os dados que emergiram do segundo encontro focal, a partir da seguinte questão disparadora: Quais os cuidados de enfermagem são implementados na assistência aos indivíduos portadores de feridas? O registro dos dados foi efetuado mediante gravação em áudio, e por um observador não participante, formando o conjunto de dados analisados.

0 tratamento dos dados foi realizado a partir da análise temática, seguindo as etapas de pré-análise, de exploração do material e de tratamento dos resultados/inferência/ interpretação. Na pré-análise foi realizada a leitura do conjunto do material, buscando a apreensão das particularidades e a percepção do conjunto de dados. $\mathrm{Na}$ etapa de exploração do material, os dados foram classificados e codificados em categorias temáticas. Na última etapa, os dados foram interpretados de acordo com o referencial teórico adotado e outros achados em pesquisas acerca do tema. ${ }^{7}$

O presente estudo segue as perspectivas éticas preconizadas na Resolução 196/96, que regulamenta as pesquisas envolvendo seres humanos. Obteve aprovação do Comitê de Ética em Pesquisa da Universidade Federal do Pampa (UNIPAMPA), sob o número 0342011. A participação dos enfermeiros foi oficializada, mediante a assinatura do Termo de Consentimento Livre e Esclarecido, que forneceu esclarecimentos e informações acerca da metodologia e objetivos da pesquisa. Os depoimentos dos participantes foram identificados com a sigla $E$, seguida de um número aleatório ( $E 1, E 2, \ldots \ldots . E 8)$.

\section{RESULTADOS E DISCUSSÃO}

Considerando as discussões entre os enfermeiros participantes do estudo, foi possível conhecer as tecnologias implementadas no cuidado aos indivíduos portadores de ferida, no contexto da atenção primária em saúde. Foram estruturadas três categorias temáticas: comunicação e vínculo na mediação do cuidado aos indivíduos portadores de feridas; sistematização da assistência de enfermagem aos indivíduos portadores de feridas; e terapias tópicas no cuidado aos indivíduos portadores de feridas.

\section{Comunicação e vínculo na mediação do cuidado aos indivíduos portadores de feridas}

Nas discussões entre os enfermeiros, a comunicação e o vínculo foram destacadas como as principais tecnologias leves presentes no processo de cuidar em enfermagem ao indivíduo portador de ferida. A comunicação e o vínculo precisam ser estabelecidos para que seja possível realizar ações eficazes de educação em saúde, em especial as orientações para a promoção do autocuidado.

A gente tem que orientar o que é importante. Para isso, é preciso manter o vínculo e a comunicação. (E1)

Nós orientamos o paciente para o autocuidado. Mas a orientação só é efetiva se tiver essa aproximação, conversa, escuta [...]. (E2)

Dessa forma, observa-se que ao resgatar essas tecnologias no cuidado aos portadores de feridas, apresenta-se uma possibilidade para que esses indivíduos sejam também agentes ativos na produção de sua saúde. Ampliar o olhar e a escuta e possibilitar que a complexidade da vida dos usuários invada as unidades de saúde e a forma como os trabalhadores compreendem o processo de saúde e doença, também implica em situar esse indivíduo na posição central desse processo. ${ }^{9}$ 


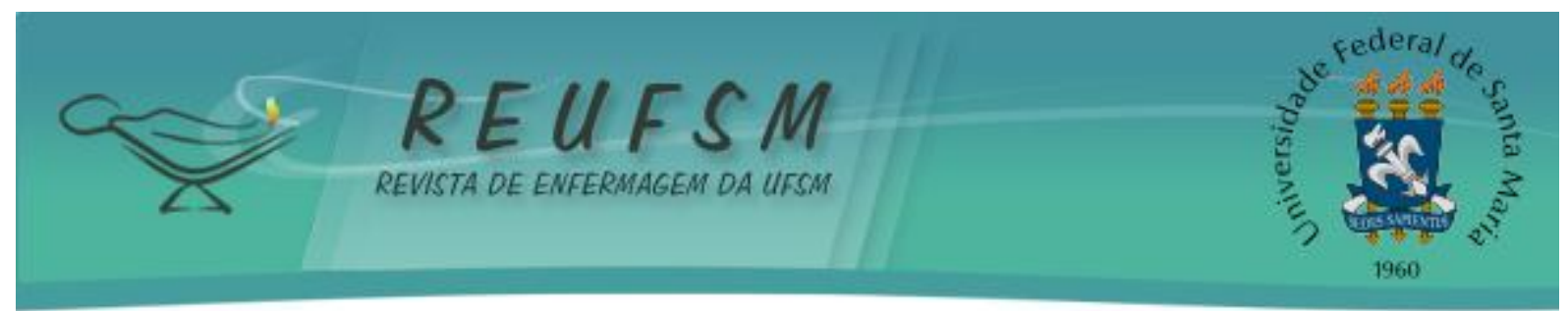

Conforme os enfermeiros, observa-se que o vínculo desse usuário com o serviço e com os profissionais da saúde é estabelecido a partir do sentimento de segurança. Ademais, os indivíduos portadores de feridas são contagiados por um sentimento de confiança que permite a aproximação dos profissionais e a formação de um vínculo com o serviço de saúde.

Fazer com que ele se sinta seguro, todas às vezes que ele precisar do serviço da unidade. (E3)

Ele vai uma vez. Na segunda ele faz certo. Na terceira ele vai tomando a liberdade de perguntar, me deixando ajudar em todas as coisas. (E4)

Quando ele tem confiança e sabe que pode receber ajuda de quem estiver na unidade básica, ele sente-se seguro e retorna. (E5)

A comunicação e o vínculo são fundamentais para que os indivíduos portadores de feridas sejam reconhecidos como sujeitos com desejos, valores, saberes e potenciais. Esses aspectos são imprescindíveis para a compreensão da singularidade vivida pelo usuário, possibilitando a construção de um plano de cuidados adequado. Nesse sentido, as orientações se condicionam como ações prioritárias de cuidado que visam, principalmente, o autocuidado. ${ }^{9}$

\section{Sistematização da assistência de enfermagem aos indivíduos portadores de feridas}

Conforme debate entre os enfermeiros, a partir da sistematização da assistência de enfermagem, são introduzidas tecnologias leve-duras nas ações de cuidado aos indivíduos portadores de feridas. Observa-se que, na atenção primária, o processo de enfermagem é destacado como uma metodologia assistencial para o cuidado neste contexto.

\section{É importante a avaliação da equipe de enfermagem. (E1)}

A avaliação é um dos cuidados mais importantes, sem dúvida. (E3)

No primeiro momento, realizamos uma anamnese para diagnosticar possíveis problemas que englobam a situação. (E6)

0 processo de enfermagem foi identificado como uma tecnologia leve-dura presente no cuidado ao indivíduo portador de feridas na atenção primária. Essa metodologia assistencial envolve um conhecimento estruturado que tem por objetivo implementar ações sistematizadas e inter-relacionadas para organizar e priorizar o cuidado, a partir de cinco etapas: investigação, diagnóstico, planejamento, intervenções e avaliação dos cuidados. ${ }^{10}$

0 histórico de enfermagem, também nomeado como investigação, se constitui como uma das etapas do processo de enfermagem mais utilizadas na assistência ao portador de ferida. A partir da avaliação da história de saúde e da lesão, o enfermeiro pode elaborar um plano de cuidados que atenda as necessidades desse indivíduo, que vão além dos cuidados dedicados à lesão tecidual. É preciso avaliar a condição clínica e considerar que ele enfrenta diversas situações, que também podem afetar seu estado emocional e a sua condição sociocultural. ${ }^{11}$

A partir dessa avaliação, são definidos os diagnósticos que permeiam o processo de cuidar. Para tanto, é importante identificar as necessidades dos indivíduos ${ }^{9}$, utilizando o 


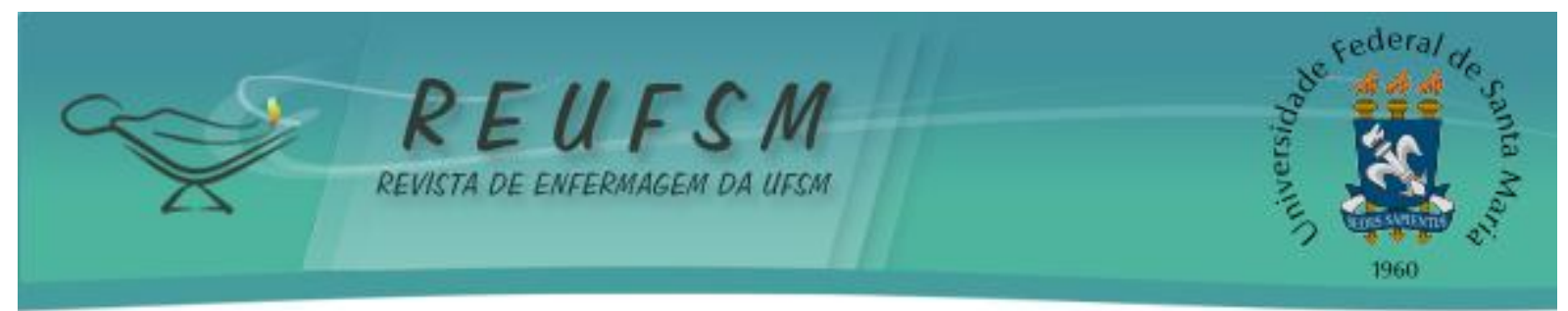

pensamento crítico e o raciocínio clínico como instrumentos para operar essa tecnologia levedura de cuidado. O raciocínio clínico está presente em todas as ações e decisões assistenciais do enfermeiro: no diagnóstico das necessidades de saúde e de doença, na escolha de intervenções apropriadas e na avaliação dos resultados obtidos. ${ }^{12-13} 0$ pensamento crítico é definido como uma habilidade mental e cognitiva necessária para o desenvolvimento do raciocínio clínico. Com esses conhecimentos já existentes, é possível que o enfermeiro identifique as normalidades e anormalidades orgânicas, e sua relação com o estado clínico do paciente. ${ }^{12}$

As tecnologias leves-duras, principalmente aquelas relacionadas à avaliação do indivíduo portador de ferida, precisam embasar os processos de trabalho em saúde, com o intuito de produzir o cuidado em enfermagem centrado nos usuários, incluindo, além da doença, o sujeito em seu contexto coletivo. Nesse sentido, o objeto de atenção, os meios e as finalidades que envolvem a produção do cuidado, visam à cura e o alívio do sofrimento, bem como o desenvolvimento da autonomia dos indivíduos para assumirem seus problemas e condições concretas de vida. ${ }^{14-15}$

Outra característica identificada em relação às tecnologias leves-duras foi à necessidade de complementaridade de saberes e campos de atuação, dos diferentes profissionais da equipe, no sentido de proporcionar um cuidado integral. Os enfermeiros participantes destacaram a importância do trabalho multiprofissional, com o objetivo de promover o suporte psicológico e social ao indivíduo. Assim, ressaltam que é de responsabilidade do enfermeiro identificar a necessidade do acompanhamento paralelo de outros profissionais, objetivando a melhora das condições de tratamento e recuperação.

Em alguns casos, é preciso encaminhar a outros profissionais, isso também faz parte do cuidado. (E2)

Encaminhar o paciente para acompanhamento psicológico $e$ assistência social, pode ajudar muito [...]. (E3)

Temos que resgatar mais isso. 0 indivíduo que tem uma ferida precisa dessa equipe multiprofissional para ter uma assistência de qualidade. (E4)

O encaminhamento do indivíduo portador de ferida para outros profissionais que compõem a equipe multiprofissional se constitui como uma estratégia importante para complementar a assistência. Essa perspectiva, no âmbito das tecnologias leves-duras, representa a produção coletiva em saúde. ${ }^{16} \mathrm{~A}$ assistência, nas organizações de saúde, se caracteriza como multidisciplinar, isto é, depende da conjugação do trabalho de vários profissionais que atuam como operadores das tecnologias de saúde. A assistência idealizada, recebida e vivida por todos os sujeitos envolvidos, é o somatório de um grande número de ações parciais que vão se complementando, de maneira mais ou menos consciente e negociada, entre os vários profissionais. ${ }^{11}$

\section{Terapias tópicas no cuidado aos indivíduos portadores de feridas}

As tecnologias duras que permeiam o cuidado ao indivíduo portador de feridas envolvem os dispositivos, materiais e coberturas farmacológicas utilizadas na terapia tópica. Em relação a essas tecnologias duras, os participantes demonstraram preocupação com disponibilidade desses recursos necessários para implementar os cuidados, a partir da 


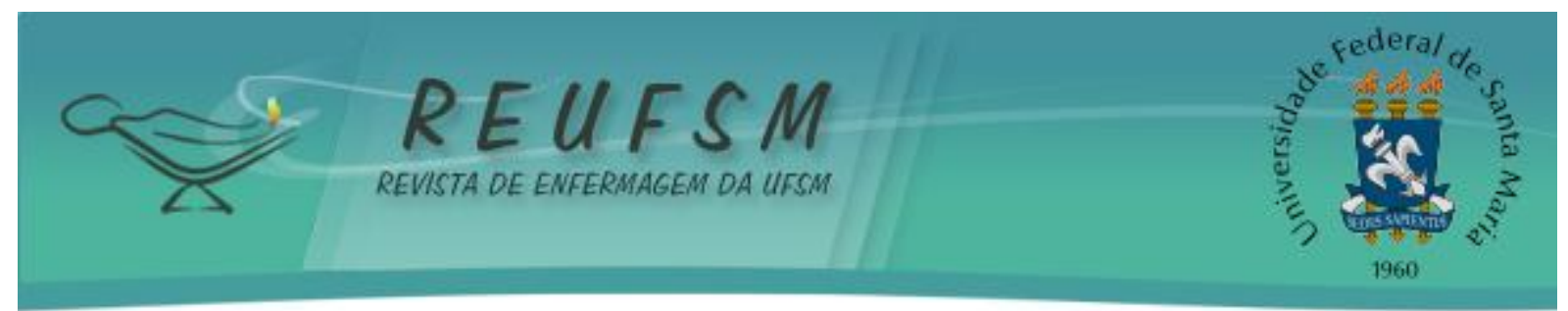

realização do curativo. Ademais, foi evidenciada a importância dos exames laboratoriais e periódicos, também considerados como uma tecnologia dura.

Nós precisamos do kit de materiais e de curativo para fazer a assistência. (E5)

E a nossa atenção também está voltada para a disponibilidade desses materiais, coberturas e tudo que for necessário para fazer um curativo adequado. $O$ cuidado para este indivíduo também é isso. (E3)

Além disso, também nos preocupamos com os exames periódicos. Precisamos buscar e orientar esse cuidado, que assim como os materiais nem sempre estão disponíveis. (E8)

As tecnologias duras de cuidado são representadas pelos materiais concretos, tais como os equipamentos tecnológicos, normas e estruturas organizacionais. ${ }^{9} \mathrm{Na}$ assistência ao indivíduo portador de ferida, evidenciou-se que os enfermeiros conferem importância aos materiais utilizados na realização dos cuidados diretos com a lesão tecidual, e aos exames laboratoriais para acompanhamento.

No contexto da atenção primária, cenário investigativo do estudo, espera-se que predominem as tecnologias leves e leves-duras, em relação às tecnologias duras. Todavia, na maioria dos serviços de saúde, a atenção primária ainda permanece ligada ao recurso instrumental. Assim, questiona-se: por que o cuidado ao indivíduo portador de ferida, mesmo na atenção primária, ainda permanece vinculado apenas à realização do curativo?

Essa perspectiva sugere que, em alguns âmbitos de cuidado, prevalece um modo de produção de saúde que enfatiza o uso de tecnologias duras, em detrimento de tecnologias leve-duras e leves. Assim, o cuidado de enfermagem ao indivíduo portador de ferida na atenção primária e nos demais âmbitos assistências, frequentemente, fica restrito ao momento do curativo, ou seja, as ações de cuidado direcionadas a lesão tecidual. Na produção científica, de igual forma, essa perspectiva é visualizada, pois a maioria das pesquisas desenvolvidas acerca dessa temática está direcionada a tecnologias duras de cuidado, envolvendo coberturas farmacológicas e outros procedimentos técnicos.

A ênfase nas tecnologias duras é resultado do modelo hegemônico vigente na área da saúde. Nesse sentido, a baixa resolutividade na atenção primária pode estar relacionada ao exercício hegemônico de uma clínica centrada no ato prescritivo e na produção de procedimentos, em ação substitutiva a prática que valoriza a clínica como o exercício ampliado de múltiplos profissionais. ${ }^{9}$

Para a superação desse modelo assistencial, é necessária uma inversão das tecnologias de cuidado a serem utilizadas na produção da saúde. Os processos de trabalho surgem como os principais alvos das mudanças nos serviços de saúde, no sentido de reestruturar e organizar as ações com ênfase no usuário e suas necessidades. ${ }^{9}$ Com isso, a produção de cuidados aos indivíduos portadores de feridas se torna um território no qual deve predominar as tecnologias leves, o que permite o desenvolvimento de novas estratégias para a construção de novos saberes, compreensões e relações.

Outros aspectos importantes relacionados às tecnologias duras envolvem o desenvolvimento e aprimoramento de habilidades relacionadas ao uso de materiais e equipamentos específicos para o cuidado do indivíduo portador de ferida, como por exemplo, o uso de bolsas de colostomias e coberturas farmacológicas. Segundo os enfermeiros, o conhecimento científico, relacionado a esses dispositivos de cuidado precisa ser atualizado, a partir de capacitações e aperfeiçoamento dos profissionais da saúde. 


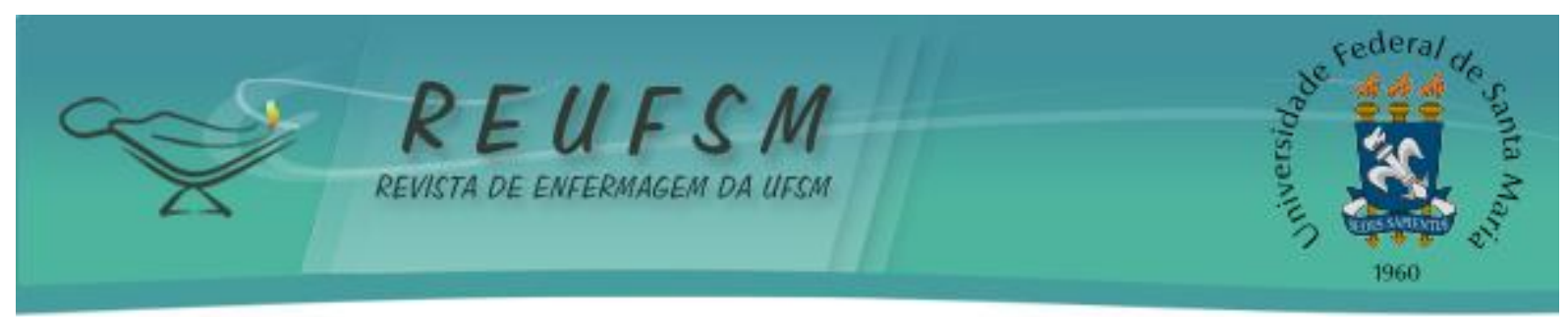

As trocas da colostomia, por exemplo, precisam de um treinamento que é realizado na unidade básica. (E7)

Realiza-se a higiene, coloca-se o pó regenerador, o qual fica agindo embaixo de uma película protetora. (E1)

Tem as dificuldades encontradas para utilizar algumas coberturas [...]. (E3)

Observa-se a preocupação dos enfermeiros com as coberturas farmacológicas. É importante destacar que não há uma única cobertura adequada para todas as feridas. É importante considerar às especificidades de cada tipo ou fase das lesões. Nesse sentido, os enfermeiros precisam conhecer e testar novas tecnologias, com o objetivo de possibilitar a argumentação junto aos gestores e demais profissionais da equipe de saúde sobre a necessidade de disponibilidade das coberturas. Todavia, o tratamento não depende só da aplicação de agentes tópicos e coberturas, é preciso realizar avaliação interdisciplinar do usuário e agregá-lo no plano terapêutico, especialmente nos casos de feridas crônicas. ${ }^{17}$

De um modo geral, os aperfeiçoamentos oportunizados aos profissionais acerca dessa temática, envolvem as inovações acerca dos tipos de coberturas farmacológicas e outros procedimentos técnicos. Nesse sentido, observa-se a necessidade de criação de espaços coletivos para a reflexão acerca do processo de trabalho ${ }^{9}$, com o objetivo de compartilhar as diferentes concepções e atuações no conjunto da assistência desenvolvida ao indivíduo portador de ferida. É também uma oportunidade para conhecer o valor que cada profissional tem nesse processo, possibilitando que todos possam repensar suas ações e inovar suas práticas a partir dos novos elementos que são compartilhados e construídos nesse coletivo.

\section{CONSIDERAÇÕES FINAIS}

No âmbito dos cuidados de enfermagem aos indivíduos portadores de feridas é imprescindível a implementação das tecnologias leves, leve-duras e duras. A partir do presente estudo, evidencia-se a preocupação dos enfermeiros com as tecnologias leves e leve-duras. Esse fato demonstra que esses profissionais estão dispostos a aprimorar seus conhecimentos. Ademais, destaca-se a importância da formação do vínculo e da confiança, que se constituem como tecnologias leves de cuidado, indispensáveis para alcançar a resolutividade e qualidade do cuidado de enfermagem.

A visão das enfermeiras, em relação às tecnologias duras, é restrita ao campo material sendo relacionada, principalmente, a troca de curativos e aos encaminhamentos para exames laboratoriais. Os saberes estruturados também são uma tecnologia dura, por exemplo, a utilização de protocolos para o atendimento a essa população. Um protocolo específico pode contribuir positivamente na qualificação a atuação dos profissionais de enfermagem no cuidado aos portadores de feridas.

Enfim, entende-se que o presente estudo poderá contribuir significativamente para a ampliação da visão das participantes sobre o atendimento ao indivíduo portador de feridas, qualificando o cuidado de enfermagem. Além disso, este estudo possibilita a aproximação e interação entre academia e as instituições de saúde, consolidando parcerias e firmando espaços para o desenvolvimento de atividades de ensino, pesquisa e extensão.

\section{REFERÊNCIAS}

1. Martins CR, Dal Sasso GTM. Tecnologia: definiç̧̃̃es e reflexões para a prática em saúde e enfermagem. Texto \& Contexto Enferm. 2008;17(1):11-2. 


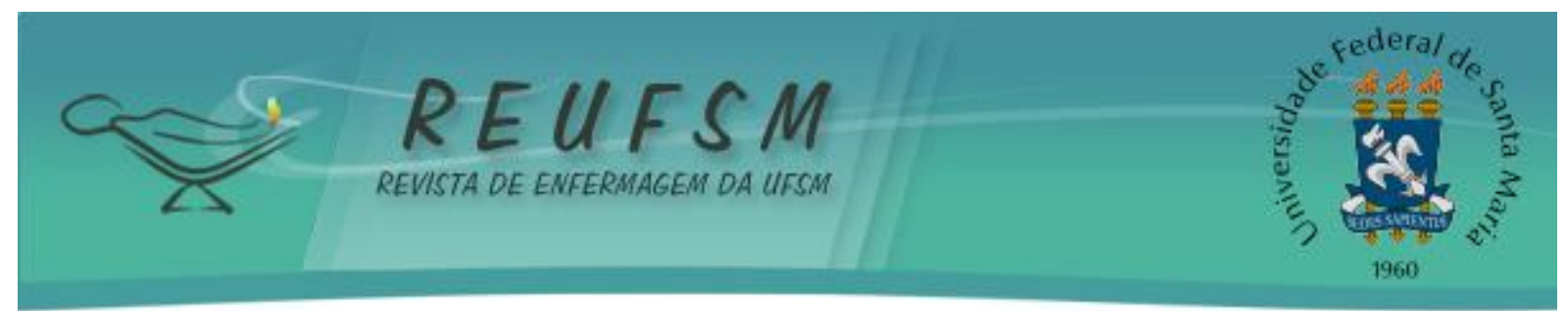

2. Silva RC, Ferreira MA. A tecnologia em saúde: uma perspectiva psicossociológica aplicada ao cuidado de enfermagem. Esc Anna Nery Rev Enferm. 2009;13(1):169-73.

3. Rocha PK, Prado ML, Wall ML, Carraro TE. Cuidado e tecnologia: aproximações através do Modelo de Cuidado. Rev Bras Enferm. 2008;61(1):113-6.

4. Lopes EM, Pinheiro AKB, Pinheiro PNC, Vieira NFC. Tecnologia e práticas de enfermagem - um estudo bibliográfico. Online Braz J Nurs [Internet]. 2009 [acesso em 2013 maio 15];8(1). Disponível em: http://www.objnursing.uff.br/index.php/nursing/article/view/j.16764285.2009.1883/446.

5. Merhy EE, Feuerwerker LCM. Novo olhar sobre as tecnologias de saúde: uma necessidade contemporânea. In: Mandarino ACS, Gomberg E, organizadores. Leituras de novas tecnologias e saúde. São Cristóvão(SE): Editora UFS; 2009.

6. Ministério da Saúde (Brasil). Secretaria de Atenção à Saúde. Departamento de Atenção Básica. Política nacional de atenção básica. Brasília (DF): Ministério da Saúde; 2006.

7. Minayo MCS, Deslandes SF, Gomes R. Pesquisa social: teoria, método e criatividade. Petrópolis: Vozes; 2010.

8. Pope C, Mays N. Pesquisa qualitativa na atenção à saúde. $3^{\mathrm{a}}$ ed. Porto Alegre: Artmed; 2009.

9. Merhy EE. Saúde: a cartografia do trabalho vivo. São Paulo: HUCITEC; 2002.

10. Alfaro-Lefevre R. Aplicação do processo de enfermagem: uma ferramenta para o pensamento crítico. Porto Alegre: Artmed; 2010.

11. Pires VMMM, Rodrigues VP, Nascimento MAA. Sentidos da integralidade do cuidado na saúde da família. Rev Enferm UERJ. 2010;18(4):622-7.

12. Tanner CA. Thinking like a nurse: a research-based model of clinical judgment in nursing. J Nurs Educ [Internet]. 2006 [acesso em 2013 jan 13];45(6):204-11. Disponível em: http://ahn.mnsu.edu/nursing/facultyformsandinfo/thinkinglikeanurse.pdf .

13. Cerulo JASB, Cruz DALM. Raciocínio clínico e pensamento crítico. Rev Latinoam Enferm [Internet]. 2010 jan/fev [acesso em 2013 fev 24];18(1). Disponível em: http://www.scielo.br/pdf/rlae/v18n1/pt_19.pdf.

14. Junges JR, Selli L, Soares NA, Fernandes RBP, Schreck M. Processos de trabalho no Programa Saúde da Família: atravessamentos e transversalidades. Rev Esc Enferm USP. 2009;43(4):937-44.

15. Matumoto S, FortunaCM, Kawata LSK, Mishima SM, Pereira MJB. A prática clínica do enfermeiro na atenção básica: um processo em construção. Rev Latinoam Enferm [Internet]. 2011 [acesso em 2012 set 25];19(1). Disponível em: http://www.scielo.br/pdf/rlae/v19n1/pt_17.pdf.

16. Cardoso GB, Silva ALA. O processo de trabalho na enfermagem: articulação das tecnologias do cuidado. Rev Enferm UERJ. 2010;18(3):451-5.

17 Silva DS, Hahn GV. Cuidados com úlceras venosas: realidade do Brasil e Portugal. Rev Enferm UFSM [Internet]. 2012[acesso em $2013 \mathrm{fev}$ 24];2(2):330-8. Disponível em: http: //cascavel.ufsm.br/revistas/ojs-2.2.2/index.php/reufsm/article/view/4967

Data de recebimento: 04/04/2013

Data de aceite: $28 / 05 / 2013$ 


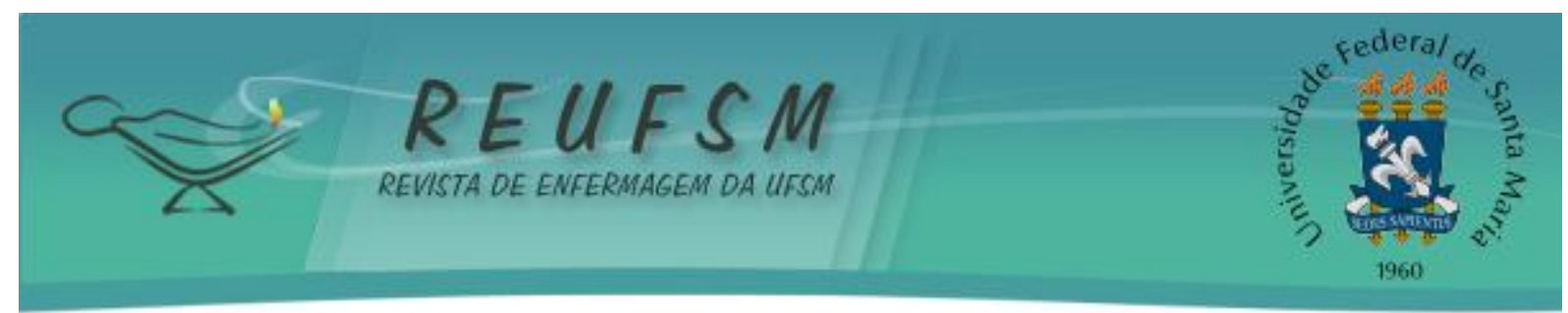

Contato com autor responsável: Josefine Busanello

Endereço: BR 472, Km 592, Caixa Postal 118. Uruguaiana/RS.

CEP: $97500-970$

E-mail: josefinebusanello@unipampa.edu.br 